\title{
Modifying the atlas of low lunar orbits using inert tethers ${ }^{\text {is }}$
}

\author{
Martin Lara ${ }^{\mathrm{a}, *}$, Jesús Peláez ${ }^{\mathrm{b}}$, Hodei Urrutxua ${ }^{\mathrm{b}}$ \\ ${ }^{a}$ Real Observatorio de la Armada, ES-11110 San Fernando, Spain \\ ${ }^{\mathrm{b}}$ Space Dynamics Group (UPM), ETSI Aeronáuticos, ES-28040 Madrid, Spain
}

Keywords:

Frozen orbits

Tethers

Dumbbell model

Lunar gravity field

Averaging

\begin{abstract}
A B S T R A C T
For long enough tethers, the coupling of the attitude and orbital dynamics may show non-negligible effects in the orbital motion of a tethered satellite about a central body. In the case of fast rotating tethers the attitude remains constant, on average, up to second order effects. Besides, for a tether rotating in a plane parallel to the equatorial plane of the central body, the attitude-orbit coupling effect is formally equal to the perturbation of the Keplerian motion produced by the oblateness of the central body and, therefore, may have a stabilizing effect in the orbital dynamics. In the case of a tethered satellite in a low lunar orbit, it is demonstrated that feasible tether lengths can help in modifying the actual map of lunar frozen orbits.
\end{abstract}

\section{Introduction}

Unlike frozen orbits about Earth-like planets, for which the oblateness coefficient dominates the dynamics and exists a continuous transition of near circular frozen orbits from low inclinations to polar (excluding the critical inclination) [1,2], lunar frozen orbits are restricted by the distribution of the lunar mascons and Earth-Moon geometry perturbations. The Moon being less flattened than Earth, its oblateness coefficient does not play a dominant role in a gravitational field in which its lumpy character confers similar importance to a high number of harmonics [3,4].

Hence, the unique gravitational features of the Moon prevent the existence of low lunar frozen orbits except for a handful of narrow regions of inclination. In addition, the eccentricity of the frozen orbits is far from circular except for the known inclinations of $\sim 27^{\circ}, 50^{\circ}, 76^{\circ}$ and $86^{\circ}[3,5]$.

\footnotetext{
Part of this research was presented in the Global Lunar Conference, Beijing (China), May 31-June 3, 2010.

* Corresponding author. Tel.: +34856119449.

E-mail addresses: mlara0@gmail.com, mlara@roa.es (M. Lara), j.pelaez@upm.es (J. Peláez), hodei.urrutxua@upm.es (H. Urrutxua).
}

This fact makes that common lunar mapping missions requiring near circular orbits in polar inclinations need of orbit maintenance maneuvers to keep the spacecraft within a desired altitude range, with the consequent effect on the duration of the mission. Then, the usefulness of the frozen orbit architecture is normally being limited to the commissioning period [6].

However, a great amount of control can be avoided with a new concept, namely using fast rotating inert tethers $[7,8]$. While orbital and attitude dynamics decouple for usual artificial satellites, the coupling between orbital and rotational motion is not negligible in the case of long enough tethers. Indeed, as it has been demonstrated for Halo orbits, this attitude-orbit coupling introduces a perturbation that, despite being small, brings noticeable changes into the shape of the orbit, and may even be enough to change the orbit's stability character $[7,8]$.

In the case of rotating tethers the centrifugal forces generated by the tether's angular velocity increase the tether's tension, which is the stabilizing element of the flexible tether; therefore, the dumbbell model is very appropriate for approximating the tether's dynamics. In this approximation, we find a particular attitude configuration whose coupling with the orbital dynamics may 
counterbalance the central body's oblateness perturbation. However, this conspicuous configuration is not expected to be held without the help of active control and, therefore, it may be considered anecdotic. Nevertheless, this motivates the search for other configurations with relevant mechanical implications in the orbital dynamics about oblate bodies.

We focus on the case of fast rotating inert tethers, or FRITs. When the tether's rotation period is much shorter than its orbital period, the relevant dynamics of the FRIT can be studied by averaging the tether's rotation angle. On average, up to the second order terms in the ratio of the orbital mean motion to the tether's self rotation rate, the FRIT's attitude remains constant. Furthermore, we demonstrate that when the FRIT's rotation takes place in a plane parallel to the equatorial plane of the central body, the relevant orbital dynamics is formally equivalent to the main problem of Artificial Satellite Theory (when only the zonal $J_{2}$ is retained in the perturbation). In this scenario, a tether's characteristic length appears as an analog of the (dimensional) oblateness coefficient. This characteristic length depends only on the physical length of the inert tether, which we may change at our will, and the mass distribution of the tethered satellite, a design parameter. Hence, a tethered satellite can be designed to modify artificially the oblateness effect of a central body.

An immediate application of the FRIT concept is found for producing a virtual increase in the oblateness of the Moon. Thus, it is shown that the Moon's second order zonal coefficient is effectively doubled when using a FRIT with a length of several tens of $\mathrm{km}$. This virtual increase of the oblateness makes the Moon's $J_{2}$ coefficient more dominant over other perturbations due to the non-centrality of the gravitational field, and hence introduces radical changes in the shape and distribution of low lunar frozen orbits. As a general rule, low lunar frozen orbits are found with lower eccentricities now, and the range of frozen orbits is extended to wider regions of inclinations. Thus, for instance, we find that the eccentricity of the frozen orbit that has been used for the commissioning period of the Lunar Reconnaissance Orbit [6] can be reduced by half when using a FRIT of $40 \mathrm{~km}$ length.

\section{Inert tether dynamics}

We consider a tethered satellite in the dumbbell model approximation. That is, a theoretical model made of two end masses joined by an inert rigid rod of negligible mass, the length of the rode being large when compared with the dimensions of the end masses, c.f. the illustration in Fig. 1. Throughout the paper, we will call this model a tethered satellite, or simply the tether when there is no risk of confusion.

We only take into account the gravitational forces acting on the inert tether. Therefore, we deal solely with the rigid-body dynamics of the tether attracted by a central body. Details on the derivation of the relevant dynamic equations can be found in Refs. [7,8] that we summarize here for completeness.

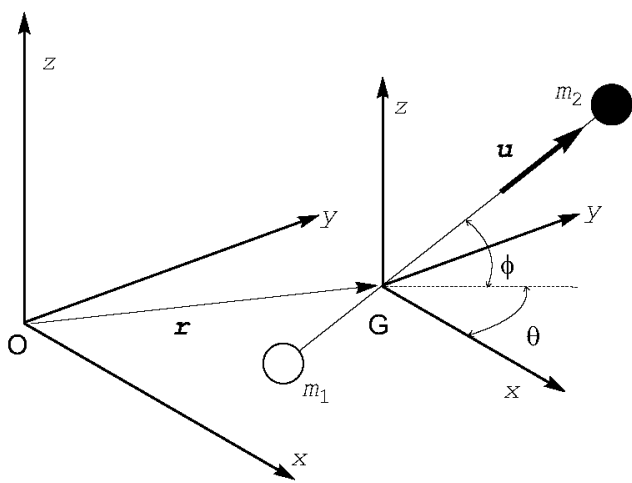

Fig. 1. Tether satellite in the inertial frame. The pitch $\phi$ and roll $\theta$ angles describe the tether's attitude in the inertial frame.

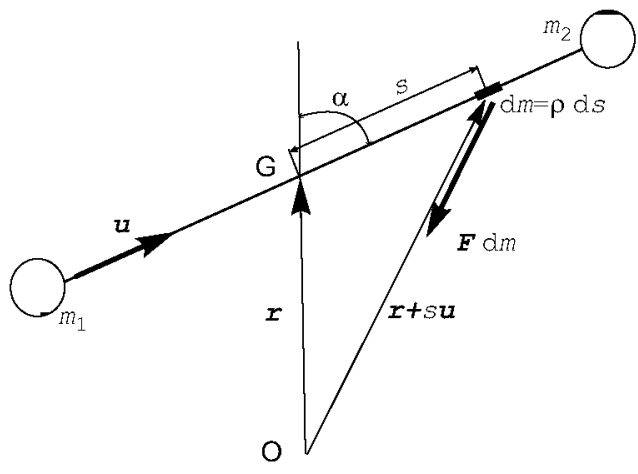

Fig. 2. Dumbbell model kinetics.

The problem is conservative and the total energy is made of the kinetic energy plus the potential one

$E=\frac{1}{2} m\left(\frac{\mathrm{d} \mathbf{r}}{\mathrm{d} t} \cdot \frac{\mathrm{d} \boldsymbol{r}}{\mathrm{d} t}\right)+\frac{1}{2} \boldsymbol{\Omega} \cdot \mathbf{I} \cdot \mathbf{\Omega}+V$

where $m$ is the total mass of the tethered satellite, $\mathbf{I}$ is the central inertia tensor, $\boldsymbol{r}$ is the position vector of the tether's center of mass $G, \boldsymbol{\Omega}$ is the tether's rotation vector, and the potential energy $V$ is to be determined.

\subsection{Orbital motion}

The inertial acceleration of the center of mass of a tethered satellite under the action of a central body is

$\frac{\mathrm{d}^{2} \boldsymbol{r}}{\mathrm{d} t^{2}}=-\nabla V$

The potential energy is

$V=-\int_{m} \frac{\mu}{\|\varrho\|} \mathrm{d} m$

where $\mu$ is the gravitational parameter of the attracting body and $\varrho$ is the position vector of the mass element. Then, in reference to Fig. 2

$V=-\int_{m} \frac{\mu}{\sqrt{r^{2}+2 r s \cos \alpha+s^{2}}} \mathrm{~d} m$

where $r=\|\boldsymbol{r}\|, s$ is the distance from $G$ to the element of differential of mass of density $\rho, \mathrm{d} m=\rho \mathrm{d} s$, the angle $\alpha$ is 
defined from $\cos \alpha=\boldsymbol{u} \cdot \hat{\boldsymbol{r}}$, "hats" mean unit vectors, and the unit vector $\boldsymbol{u}$ takes the direction defined by the tether's length.

In practical applications $s$ is small when compared with $r$, and the potential is developed by the usual expansion in Legendre polynomials $P_{n}$

$V=-\frac{\mu}{r} \sum_{n \geq 0}\left(\frac{\lambda}{r}\right)^{n} a_{n} P_{n}(\cos \alpha)$

where $\lambda$ is the tether's physical length, and the nondimensional coefficients $a_{n}$ are function of the tether mass $m_{T}$ and end masses $m_{1}$ and $m_{2}$ (cf. Appendix $C$ of Ref. [7]).

If we work at second order of $\lambda / r$, we get

$V=-m \frac{\mu}{r}\left[1+\frac{\lambda^{2}}{r^{2}} a_{2} P_{2}(\boldsymbol{u} \cdot \hat{\boldsymbol{r}})+\mathcal{O}(\lambda / r)^{3}\right]$

where

$a_{2}=\frac{12 m_{1} m_{2}+4\left(m_{1}+m_{2}\right) m_{T}+m_{T}^{2}}{12\left(m_{1}+m_{2}+m_{T}\right)^{2}}, \quad 0<a_{2}<1 / 4$

For a tether of negligible mass the highest value is $a_{2}=1 / 4$, which corresponds to a tethered satellite with equal end masses. The other extreme value $a_{2}=0$ corresponds to a tether with the total mass concentrated in one of the end masses.

Therefore, the orbital equations of motion are

$\frac{\mathrm{d}^{2} \boldsymbol{r}}{\mathrm{d} t^{2}}=-\frac{\mu}{r^{3}} \boldsymbol{r}+\boldsymbol{A}$

where neglecting terms of the order of $(\lambda / r)^{3}$ and higher,

$\boldsymbol{A}=-\frac{\mu}{r^{2}} \frac{3}{2} a_{2} \frac{\lambda^{2}}{r^{2}}\left\{\left[5(\boldsymbol{u} \cdot \hat{\boldsymbol{r}})^{2}-1\right] \hat{\boldsymbol{r}}-2(\boldsymbol{u} \cdot \hat{\boldsymbol{r}}) \boldsymbol{u}\right\}$

\subsection{Attitude motion}

The attitude dynamics is described by the time evolution of the angular momentum vector $\boldsymbol{H}$. The attitude equations are

$$
\begin{aligned}
\frac{\mathrm{d} \boldsymbol{H}}{\mathrm{d} t} & =\boldsymbol{M}=\int_{m} s \boldsymbol{u} \times \mathrm{d} \boldsymbol{F}_{m} \\
& =-\frac{\mu}{r} \boldsymbol{u} \times \hat{\boldsymbol{r}} \int_{m} \frac{(s / r) \mathrm{d} m}{\left[1+2(s / r) \cos \alpha+(s / r)^{2}\right]^{3 / 2}}
\end{aligned}
$$

After the usual expansion of the denominator in power series of $s / r$, the torque $\boldsymbol{M}$ is integrated to give

$\boldsymbol{M}=m \frac{\mu}{r}(\boldsymbol{u} \times \hat{\boldsymbol{r}})\left[3 a_{2} \frac{\lambda^{2}}{r^{2}}(\boldsymbol{u} \cdot \hat{\boldsymbol{r}})+\mathcal{O}(\lambda / r)^{3}\right]$

In the system defined by the axes of maxima inertia there is null moment of inertia around $\boldsymbol{u}$, and the inertia tensor is

$\mathbf{I}=\left(\begin{array}{lll}0 & 0 & 0 \\ 0 & \mathcal{I} & 0 \\ 0 & 0 & \mathcal{I}\end{array}\right)$

where $\mathcal{I}=m a_{2} \lambda^{2}$. Then, $\boldsymbol{H}$ can be written

$\boldsymbol{H}=\mathbf{I} \cdot \boldsymbol{\Omega}=\mathcal{I} \boldsymbol{u} \times(\boldsymbol{\Omega} \times \boldsymbol{u})=\mathcal{I} \boldsymbol{u} \times \frac{\mathrm{d} \boldsymbol{u}}{\mathrm{d} t}$
Because of the symmetries of the dumbbell model we find convenience in choosing a reference system such that

$\boldsymbol{u}_{1}=\boldsymbol{u}, \quad \boldsymbol{u}_{2}=\frac{\mathrm{d} \boldsymbol{u} / \mathrm{d} t}{\|\mathrm{~d} \boldsymbol{u} / \mathrm{d} t\|}, \quad \boldsymbol{u}_{3}=\boldsymbol{u}_{1} \times \boldsymbol{u}_{2}$

Then, $\boldsymbol{H}=\mathcal{I} \Omega_{\perp} \boldsymbol{u}_{3}$, where we call $\Omega_{\perp} \equiv\|\mathrm{d} \boldsymbol{u} / \mathrm{d} t\|$, and Eq. (4) results

$\frac{\mathrm{d} \boldsymbol{H}}{\mathrm{d} t}=\mathcal{I} \frac{\mathrm{d} \Omega_{\perp}}{\mathrm{d} t} \boldsymbol{u}_{3}+\mathcal{I} \Omega_{\perp} \frac{\mathrm{d} \boldsymbol{u}_{3}}{\mathrm{~d} t}=\left(\boldsymbol{M} \cdot \boldsymbol{u}_{3}\right) \boldsymbol{u}_{3}+\left(\boldsymbol{M} \cdot \boldsymbol{u}_{2}\right) \boldsymbol{u}_{2}$

Therefore, we get the attitude equations

$\frac{\mathrm{d} \boldsymbol{u}_{1}}{\mathrm{~d} t}=\Omega_{\perp} \boldsymbol{u}_{2}$

$\frac{\mathrm{d} \boldsymbol{u}_{3}}{\mathrm{~d} t}=\frac{\boldsymbol{M} \cdot \boldsymbol{u}_{2}}{\Omega_{\perp} \mathcal{I}} \boldsymbol{u}_{2}$

$\frac{\mathrm{d} \Omega_{\perp}}{\mathrm{d} t}=\frac{\boldsymbol{M} \cdot \boldsymbol{u}_{3}}{\mathcal{I}}$

with the constrains $\left\|\boldsymbol{u}_{1}\right\|=\left\|\boldsymbol{u}_{3}\right\|=1, \boldsymbol{u}_{1} \cdot \boldsymbol{u}_{3}=0$ that reduce the dimension of the system to 4 . The explicit reduction of the dimension of the differential system when using these constrain will be shown later (see Eqs. (11)-(14) below).

\subsection{Lengthening and flattening}

Note the striking analogy between Eq. (3) and the gravitational acceleration $\boldsymbol{a}$ of a particle caused by the oblateness of a central body

$\frac{\mathrm{d}^{2} \boldsymbol{R}}{\mathrm{d} t^{2}}=-\frac{\mu}{r^{3}} \boldsymbol{r}+\boldsymbol{a}$

$\boldsymbol{a}=+\frac{\mu}{r^{2}} \frac{3}{2} J_{2} \frac{\alpha^{2}}{r^{2}}\left\{\left[5(\boldsymbol{k} \cdot \hat{\boldsymbol{r}})^{2}-1\right] \hat{\boldsymbol{r}}-2(\boldsymbol{k} \cdot \hat{\boldsymbol{r}}) \boldsymbol{k}\right\}$

where $\alpha$ is the equatorial radius of the central body, $J_{2}$ its oblateness coefficient, and the unit vector $\boldsymbol{k}$ has the direction of the symmetry axis of the oblate body.

If the tether's length is in the direction of $\boldsymbol{k}$, then the orbital acceleration due to the tether physical length subtracts directly to the acceleration caused by the oblateness of the central body. Therefore, assumed the effect of the oblateness on the distributed mass of the tether to be negligible, if we are able to maintain a nonrotating tether with this constant direction $\boldsymbol{u}=\boldsymbol{k}$ along the orbital motion, then we have an artificial way of varying (to some extent) the oblateness effect of a natural body.

However, if the tether is long enough to have an effect in the orbital dynamics, we cannot consider, in general, that the attitude dynamics decouples from the orbital one. Therefore, Eqs. (2) and (3) are only part of the problem of the roto-translational motion of the tether in a central force field.

It seems unlikely that the tether will evolve in the special configuration above without active control. Nevertheless, the similitude of Eqs. (3) and (7) motivates us to search for other possibilities in which both equations may be added directly, thus, producing the effect of a virtual modification of the gravitational characteristics of the central body, which leads us to the case of rotating tethers. 


\section{Rotating tethers}

We investigate the case of rotating tethers that evolve close to a plane parallel to the orbital plane of the primaries. For describing the attitude in this specific case, we found convenient to use Tait-Bryan rotations, which are a set of Euler rotations in the sequence 123 (see Fig. 3).

Then, the body frame is expressed in the inertial frame by the composition of the rotations

$\left(\boldsymbol{u}_{1}, \boldsymbol{u}_{2}, \boldsymbol{u}_{3}\right)=R_{1}\left(-\phi_{1}\right) \circ R_{2}\left(-\phi_{2}\right) \circ R_{3}\left(-\phi_{3}\right) \circ \mathbf{I}_{3}$

where $R_{1,2,3}$ are the usual rotation matrices, and $\mathbf{I}_{3}$ is the identity matrix of dimension 3 . Thus,

$\mathbf{u}_{1}=\left(\begin{array}{c}\cos \phi_{2} \cos \phi_{3}, \\ \cos \phi_{3} \sin \phi_{1} \sin \phi_{2}+\cos \phi_{1} \sin \phi_{3}, \\ \sin \phi_{1} \sin \phi_{3}-\cos \phi_{1} \cos \phi_{3} \sin \phi_{2}\end{array}\right)$

$\boldsymbol{u}_{2}=\left(\begin{array}{c}-\cos \phi_{2} \sin \phi_{3}, \\ \cos \phi_{1} \cos \phi_{3}-\sin \phi_{1} \sin \phi_{2} \sin \phi_{3} \\ \cos \phi_{3} \sin \phi_{1}+\cos \phi_{1} \sin \phi_{2} \sin \phi_{3}\end{array}\right)$

$\mathbf{u}_{3}=\left(\begin{array}{c}\sin \phi_{2} \\ -\cos \phi_{2} \sin \phi_{1} \\ \cos \phi_{1} \cos \phi_{2}\end{array}\right)$

After some algebra we arrive at the scalar equations of the attitude dynamics

$\frac{\mathrm{d} \phi_{1}}{\mathrm{~d} t}=-\frac{\boldsymbol{M} \cdot \boldsymbol{u}_{2}}{\Omega_{\perp} \mathcal{I}} \frac{\cos \phi_{3}}{\cos \phi_{2}}$

$\frac{\mathrm{d} \phi_{2}}{\mathrm{~d} t}=-\frac{\boldsymbol{M} \cdot \boldsymbol{u}_{2}}{\Omega_{\perp} \mathcal{I}} \sin \phi_{3}$

$\frac{\mathrm{d} \phi_{3}}{\mathrm{~d} t}=\Omega_{\perp}-\frac{\mathrm{d} \phi_{1}}{\mathrm{~d} t} \sin \phi_{2}$

$\frac{\mathrm{d} \Omega_{\perp}}{\mathrm{d} t}=\frac{\boldsymbol{M} \cdot \boldsymbol{u}_{3}}{\mathcal{I}}$

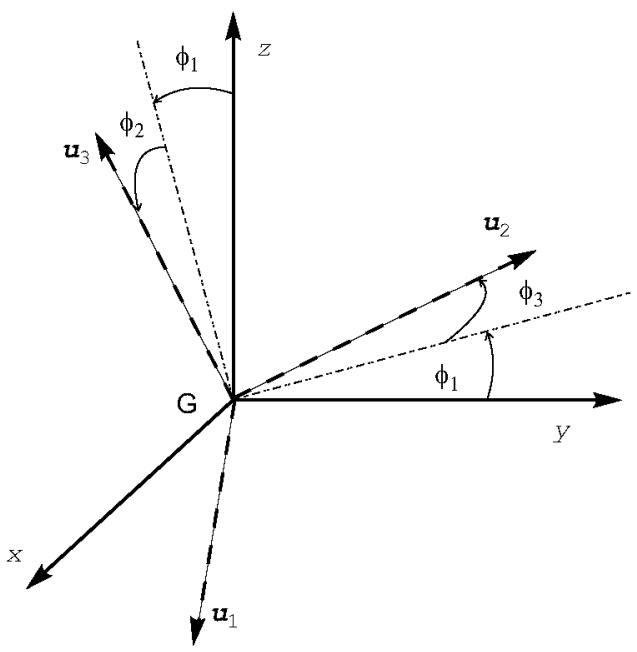

Fig. 3. Tait-Bryan rotations. where from Eqs. (5), (9) and (10),

$\boldsymbol{M} \cdot \boldsymbol{u}_{2}=3 \mathcal{I} \frac{\mu}{r^{3}} \frac{d}{r}\left(\frac{d_{1}}{r} \frac{\sin \phi_{3}}{\cos \phi_{2}}-\frac{d_{2}}{r} \cos \phi_{3}\right)$

$\boldsymbol{M} \cdot \boldsymbol{u}_{3}=\left(\boldsymbol{M} \cdot \boldsymbol{u}_{2}\right)\left(\frac{d_{1}}{d} \frac{\cos \phi_{3}}{\cos \phi_{2}}+\frac{d_{2}}{d} \sin \phi_{3}\right)$

and we introduced the auxiliary distances

$d=x \sin \phi_{2}-\left(y \sin \phi_{1}-z \cos \phi_{1}\right) \cos \phi_{2}$

$d_{1}=\left(y \cos \phi_{1}+z \sin \phi_{1}\right) \sin \phi_{2}=\frac{\partial d}{\partial \phi_{1}}$

$d_{2}=x \cos \phi_{2}+\left(y \sin \phi_{1}-z \cos \phi_{1}\right) \sin \phi_{2}=\frac{\partial d}{\partial \phi_{2}}$

that are independent from $\phi_{3}$. Then,

$\frac{\mathrm{d} \phi_{1}}{\mathrm{~d} t}=3 \frac{n^{2}}{\Omega_{\perp}} \frac{a^{3}}{r^{3}} \frac{d}{r}\left(\frac{d_{2}}{r} \frac{\cos ^{2} \phi_{3}}{\cos \phi_{2}}-\frac{d_{1}}{2 r} \frac{\sin 2 \phi_{3}}{\cos ^{2} \phi_{2}}\right)$

$\frac{\mathrm{d} \phi_{2}}{\mathrm{~d} t}=-3 \frac{n^{2}}{\Omega_{\perp}} \frac{a^{3}}{r^{3}} \frac{\mathrm{d}}{r}\left(\frac{d_{1}}{r} \frac{\sin ^{2} \phi_{3}}{\cos \phi_{2}}-\frac{d_{2}}{2 r} \sin 2 \phi_{3}\right)$

$\frac{\mathrm{d} \phi_{3}}{\mathrm{~d} t}=\Omega_{\perp}-\frac{\mathrm{d} \phi_{1}}{\mathrm{~d} t} \sin \phi_{2}=\Omega_{\perp}\left[1-\mathcal{O}\left(n / \Omega_{\perp}\right)^{2}\right]$

$$
\begin{aligned}
\frac{\mathrm{d} \Omega_{\perp}}{\mathrm{d} t}= & 3 n^{2} \frac{a^{3}}{r^{3}} \frac{d}{r}\left[\frac{1}{2}\left(\frac{1}{\cos ^{2} \phi_{2}} \frac{d_{1}^{2}}{r^{2}}-\frac{d_{2}^{2}}{r^{2}}\right) \sin 2 \phi_{3}\right. \\
& \left.-\frac{1}{\cos \phi_{2}} \frac{d_{1}}{r} \frac{d_{2}}{r} \cos 2 \phi_{3}\right]
\end{aligned}
$$

where $a$ is the orbit semimajor axis and $n=\sqrt{\mu / a^{3}}$ is the orbital mean motion.

\subsection{Fast rotating inert tether (FRIT)}

Note that, in the case of FRITs $n \ll \Omega_{\perp}$. Hence,

$\frac{\mathrm{d} \phi_{3}}{\mathrm{~d} t}=\Omega_{\perp}\left[1-\mathcal{O}\left(n / \Omega_{\perp}\right)^{2}\right] \approx \Omega_{\perp}$

and averaging over $\phi_{3}$ is, then, appropriate to focus on the long-term evolution of the system.

On average, $\Omega_{\perp}$ is constant and the attitude motion is naturally studied in a fast time scale $\tau \approx \phi_{3}$ such that $\mathrm{d} \tau / \mathrm{d} t=\Omega_{\perp}$ in which the FRIT evolves. Then,

$\frac{\mathrm{d} \phi_{1}}{\mathrm{~d} \tau}=\mathcal{O}\left(n / \Omega_{\perp}\right)^{2}, \quad \frac{\mathrm{d} \phi_{2}}{\mathrm{~d} \tau}=\mathcal{O}\left(n / \Omega_{\perp}\right)^{2}$

and, to the order of $\mathcal{O}\left(n / \Omega_{\perp}\right)^{2}$, the FRIT remains with constant attitude. Remark that the neglected second order effects mean that, in fact, the FRIT's angular momentum will slowly precess at a rate that depends on the ratio of the orbital mean motion to the FRIT's self rotation rate. If uncontrolled, this small precession will show an effect in the long-term that would even allow the FRIT's rotation plane to depart up to $90^{\circ}$ from the equatorial plane, c.f. [9]. The actual implications of these second order effects in the long-term propagation of real missions will be the topic of a future research. However, note that the rise of this precession may be delayed simply by increasing the tether's rotation, $\Omega_{\perp}$, or even cancelled by applying small control torques, which given the considerable length of 
space tethers results in a fairly inexpensive control mechanism.

With reference to the orbital motion, after replacing $\boldsymbol{u}=\boldsymbol{u}_{1}$ in Eq. (3), taking into account Eq. (8) and averaging over $\phi_{3}$, we get

$$
\begin{aligned}
\boldsymbol{A}=- & \frac{\mu}{r^{3}} \frac{a_{2}}{2} \frac{\lambda^{2}}{r^{2}}\left[3 d \left(\sin \phi_{2} \boldsymbol{i}-\cos \phi_{2} \sin \phi_{1} \boldsymbol{j}\right.\right. \\
& \left.\left.+\cos \phi_{2} \cos \phi_{1} \boldsymbol{k}\right)-\frac{3}{2}\left(5 \frac{d^{2}}{r^{2}}-1\right) \boldsymbol{r}\right]
\end{aligned}
$$

leading to the orbital equations of motion of a FRIT

$$
\begin{aligned}
& \frac{\mathrm{d}^{2} x}{\mathrm{~d} t^{2}}=-\frac{\mu}{r^{3}} x+\frac{\mu}{r^{3}} \frac{a_{2}}{2} \frac{\lambda^{2}}{r^{2}}\left[\frac{3}{2}\left(5 \frac{d^{2}}{r^{2}}-1\right) x-3 d \sin \phi_{2}\right] \\
& \frac{\mathrm{d}^{2} y}{\mathrm{~d} t^{2}}=-\frac{\mu}{r^{3}} y+\frac{\mu}{r^{3}} \frac{a_{2}}{2} \frac{\lambda^{2}}{r^{2}}\left[\frac{3}{2}\left(5 \frac{d^{2}}{r^{2}}-1\right) y+3 d \cos \phi_{2} \sin \phi_{1}\right] \\
& \frac{\mathrm{d}^{2} z}{\mathrm{~d} t^{2}}=-\frac{\mu}{r^{3}} z+\frac{\mu}{r^{3}} \frac{a_{2}}{2} \frac{\lambda^{2}}{r^{2}}\left[\frac{3}{2}\left(5 \frac{d^{2}}{r^{2}}-1\right) z-3 d \cos \phi_{2} \cos \phi_{1}\right]
\end{aligned}
$$

where $\{\boldsymbol{i} \boldsymbol{j}, \boldsymbol{k}\}$ are unit vectors of the inertial frame (cf. Fig. 1) and the tether's position is given by $\boldsymbol{r}=x \boldsymbol{i}+y \boldsymbol{j}+z \boldsymbol{k}$.

\subsection{The "oblate" configuration}

In addition, we note that for the specific configuration $\phi_{1}=\phi_{2}=0$ (see Fig. 4), then $d=z$ and the orbital equations of motion simplify dramatically to

$$
\begin{aligned}
& \frac{\mathrm{d}^{2} x}{\mathrm{~d} t^{2}}=-\frac{\mu}{r^{3}} x\left[1-\frac{\lambda^{2}}{r^{2}} \frac{3}{2} \frac{a_{2}}{2}\left(5 \frac{z^{2}}{r^{2}}-1\right)\right] \\
& \frac{\mathrm{d}^{2} y}{\mathrm{~d} t^{2}}=-\frac{\mu}{r^{3}} y\left[1-\frac{\lambda^{2}}{r^{2}} \frac{3}{2} \frac{a_{2}}{2}\left(5 \frac{z^{2}}{r^{2}}-1\right)\right] \\
& \frac{\mathrm{d}^{2} z}{\mathrm{~d} t^{2}}=-\frac{\mu}{r^{3}} z\left[1-\frac{\lambda^{2}}{r^{2}} \frac{3}{2} \frac{a_{2}}{2}\left(5 \frac{z^{2}}{r^{2}}-3\right)\right]
\end{aligned}
$$

Remarkably, if we replace $a_{2}$ by $2 J_{2}$ and $\lambda$ by $\alpha$, we obtain exactly the equations of motion of a mass-point satellite about an oblate body, Eqs. (6) and (7). Therefore,

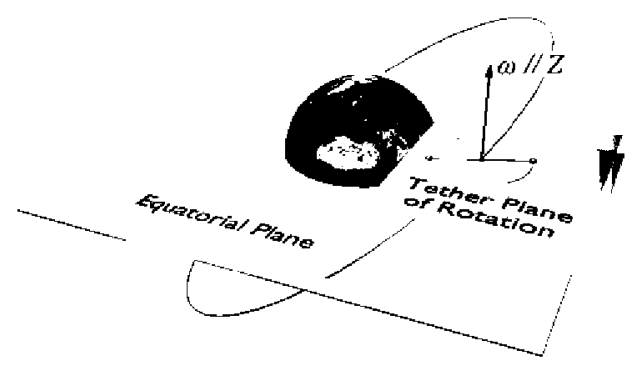

Fig. 4. The "oblate" configuration of a rotating tether $\left(\phi_{1}=\phi_{2}=0\right)$ corresponds to a FRIT whose plane of rotation remains parallel to the equator, i.e. its spin axis stays perpendicular to the equator. the dynamical effect of a FRIT lying in a plane parallel to the equatorial plane of the attracting body is to reinforce the perturbation produced by the oblateness of the attracting body. This result is quite relevant because it is well known that the oblateness perturbation may have beneficial effects in general scenarios in which other perturbations tend to destabilize the dynamics, as is the case of the motion about planetary satellites, where the gravity pull of the planet introduces instabilities in high inclination orbits $[10,11,5]$. Thus, by the simple expedient of lengthening a FRIT, we can, to some extent, mitigate instabilities induced by the dynamics.

Note that the FRIT's oblate configuration survives in the context of the restricted three-body problem $[7,8,12]$, the original proposed application of FRITs. In this case the required tether operation is to rotate fast in a plane parallel to the orbital plane of the primaries. The emphasis of previous studies, however, was put on stabilizing unstable orbits rather than in circularizing elliptic, stable orbits, as we propose below.

\section{Circularizing low lunar orbits}

By establishing the analogy between the main problem of Artificial Satellite Theory and the FRIT problem, we demonstrate that the tether's physical length $\lambda$ is a control parameter that can be used to modify the orbit characteristics of fast rotating tethers. Thus, the equation

$\lambda^{2} \frac{a_{2}}{2}=q J_{2} \alpha^{2}$

introduces a design control parameter, the tether's characteristic length

$\ell=\lambda \sqrt{\frac{1}{2} a_{2}}$

that allows to modify the FRIT's orbital characteristics in a purely dynamical way. The number $q$ quantifies the perturbing effect of the FRIT in terms of the (dimensional) oblateness coefficient of the attracting body.

These modifications of the orbit characteristics may be useful in the case of low lunar orbits, where almost circular frozen orbits are known to exist only in a handful of specific inclinations [3-5]. The conspicuous differences between the behavior of low lunar frozen orbits and Earth's or Mars's frozen orbits, for which almost circular orbits exist in all the range of inclinations except for the critical one [2], are due to the unique characteristics of the Moon's gravitational field. Since the Moon's second order zonal coefficient is notably smaller than in the case of Earth-like bodies, it does not dominate so clearly over all other harmonic coefficients. However, this unfavorable scenario for common mapping missions may be tempered to some extent by using FRITs of feasible lengths.

Fig. 5 shows the magnitude of the disturbing perturbation introduced by FRITs of different characteristic lengths for the case of a Moon orbiter, where a characteristic length of two dozens of $\mathrm{km}$ should allow for doubling the $J_{2}$ effect. Since $0<a_{2}<1 / 4$, this characteristic length would correspond to a tether's physical length $\lambda \geq 70 \mathrm{~km}$. 


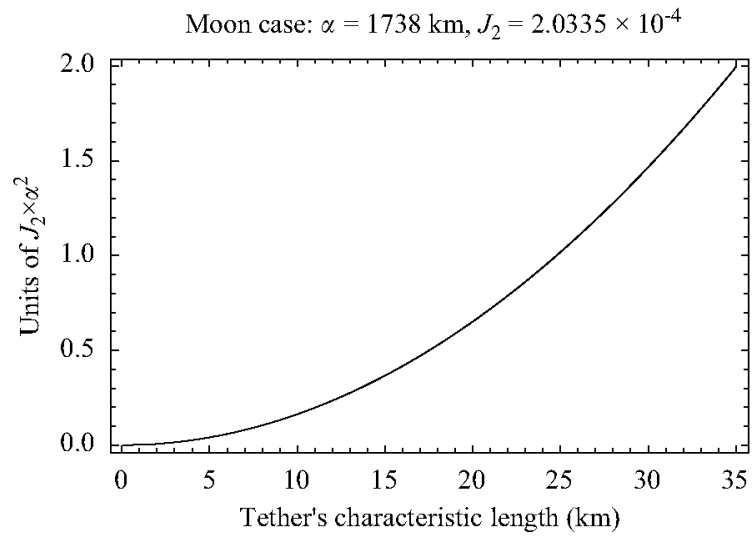

Fig. 5. Virtual effect of the FRIT's length on the Moon's oblateness.

In what follows, we assume the most favorable condition of an ideal tether of negligible mass with equal end masses. Therefore, we take a fixed $a_{2}=1 / 4$ for the remaining examples. In addition, despite satellite applications for tethers of thousands of $\mathrm{km}$ have appeared in the literature [13-17], we are interested in tether applications for low lunar orbits, say between 75 and $125 \mathrm{~km}$ over the surface of the Moon. Hence, we limit ourselves to a conservative length $\lambda=50 \mathrm{~km}$ that on one side would prevent the risk of tether impact with the lunar surface, and on the other side should be a real option for the actual characteristics of these days materials. ${ }^{1}$ For these features, we choose a tether's characteristic length $\ell=17.7 \mathrm{~km}$ a value that would strengthen the Moon's $J_{2}$ by a factor of about $50 \%$.

We recall that frozen orbits are orbits that on average have constant semimajor axis, eccentricity, inclination and argument of periapsis [18]. They are studied in the mean elements that are obtained after an averaging process that removes the fast evolving angles from the perturbing function $[19,2,20]$, although numerical searches in the non-averaged problem are also a common option [6,21,22].

The relevant equations of the frozen orbits problem are those which provide the rates of variation of the (mean) eccentricity $e$ and (mean) argument of the periapsis $\omega$. Besides, it is known that the low eccentricity frozen orbits that are of interest in common mapping satellite missions occur always with the argument of the periapsis either at $90^{\circ}$ or $270^{\circ}$. Therefore, the simple contour plot of the rate of variation of the eccentricity

$\frac{\mathrm{d} e}{\mathrm{~d} t} \equiv \dot{e}=\dot{e}\left(e, i ; \omega= \pm 90^{\circ} ; a\right)$

where $i$ is the orbital inclination, for just the specific contour that corresponds to a null rate of variation $\dot{e}=0$, will result in an inclination-eccentricity diagram of frozen orbits. These kinds of diagrams are quite useful in obtaining a global view of the frozen orbits' problem for a given

${ }^{1}$ Recall that SEDS II have successfully deployed a tether of $20 \mathrm{~km}$ in 1994, and YES2 of $32 \mathrm{~km}$ in 2007. semimajor axis (see [5], for instance), and is the tool we use for showing the effect on the frozen orbits characteristics of using FRITs.

Thus, Fig. 6 illustrates with several examples of how a FRIT with the above characteristics $\left(\lambda=50 \mathrm{~km}, a_{2}=1 / 4\right)$ modifies the atlas of low lunar frozen orbits. In this figure we show inclination-eccentricity diagrams of frozen orbits with mean semimajor axis, from top to bottom, $a=1861$, 1838 , and $1813 \mathrm{~km}$, corresponding to circular orbits at altitudes of 123,100 and $75 \mathrm{~km}$, respectively, over the lunar surface. We retained fifty zonal harmonics of the lunar potential in the disturbing function used for the computation of Eq. (15), in agreement with the usual conventions in the most rigorous literature $[3,23,24]$. The full lines correspond to the frozen orbits problem for a satellite in the mass point approximation, and the dashed curves are for a FRIT plane parallel to the equatorial plane of the Moon. In general, the curves of the FRIT case come from frozen orbits with lower eccentricities, and they exist in a wider range of inclinations than in the mass point case.

The general circularization of the frozen orbits when using FRITs is clearly noted in the case of almost polar orbits. Thus, the top plot of Fig. 6 shows that the FRIT reduces the eccentricity of the polar frozen orbit with $a=1861 \mathrm{~km}$ to $e=0.023$, which result in a periapsis height of $\approx 80 \mathrm{~km}$ about the lunar surface. Therefore, for the chosen FRIT of $50 \mathrm{~km}$ of physical length, whose ends only depart $25 \mathrm{~km}$ from the FRIT center of mass in the assumption of the equal end masses configuration, there is no risk of impact with the lunar surface.

In the center plot of Fig. $6(a=1838 \mathrm{~km})$ we see that there are not polar (non-impact) frozen orbits in the case of a mass point satellite, but we find a polar frozen orbit with $e=0.028$ in the FRIT case. Now the periapsis distance of the polar frozen orbit to the lunar surface is only of $\approx 49 \mathrm{~km}$, which also gives a safe margin for the FRIT's operation.

Finally, the FRIT polar frozen orbit in the bottom plot of Fig. 6 has an eccentricity $e=0.036$ with a semimajor axis $a=1813 \mathrm{~km}$, resulting in a periapsis distance of only about $10 \mathrm{~km}$. Although the periapsis distance is clearly shorter than the distance of the FRIT's ends to the FRIT's c.o.m., there is not risk at all of impact of the FRIT with the lunar surface at the periapsis because it occurs at $\omega=-\pi / 2$ and the FRIT is always rotating parallel to the equatorial plane of the Moon. Fig. 7 shows the minimum impact distance $\Delta$ from the FRIT to the lunar surface defined as

$\Delta=\eta-\sqrt{\alpha^{2}-\xi^{2}}-\frac{\lambda}{2}$

where $\xi=a(\cos A-e)$ and $\eta=a \sqrt{1-e^{2}} \sin A$ are the cartesian coordinates of the ellipse referred to the focus, and $A$ is the eccentric anomaly. With the definition in Eq. (16), the minimum impact distance of the polar frozen orbit is $A=11.36 \mathrm{~km}$ and occurs for $E=29.9^{\circ}$.

Last, we note that the inclination-eccentricity diagrams of Fig. 6 show only the case prograde orbits. The case of retrograde inclination frozen orbits is obtained as a reflection of these diagrams because of the inclination 

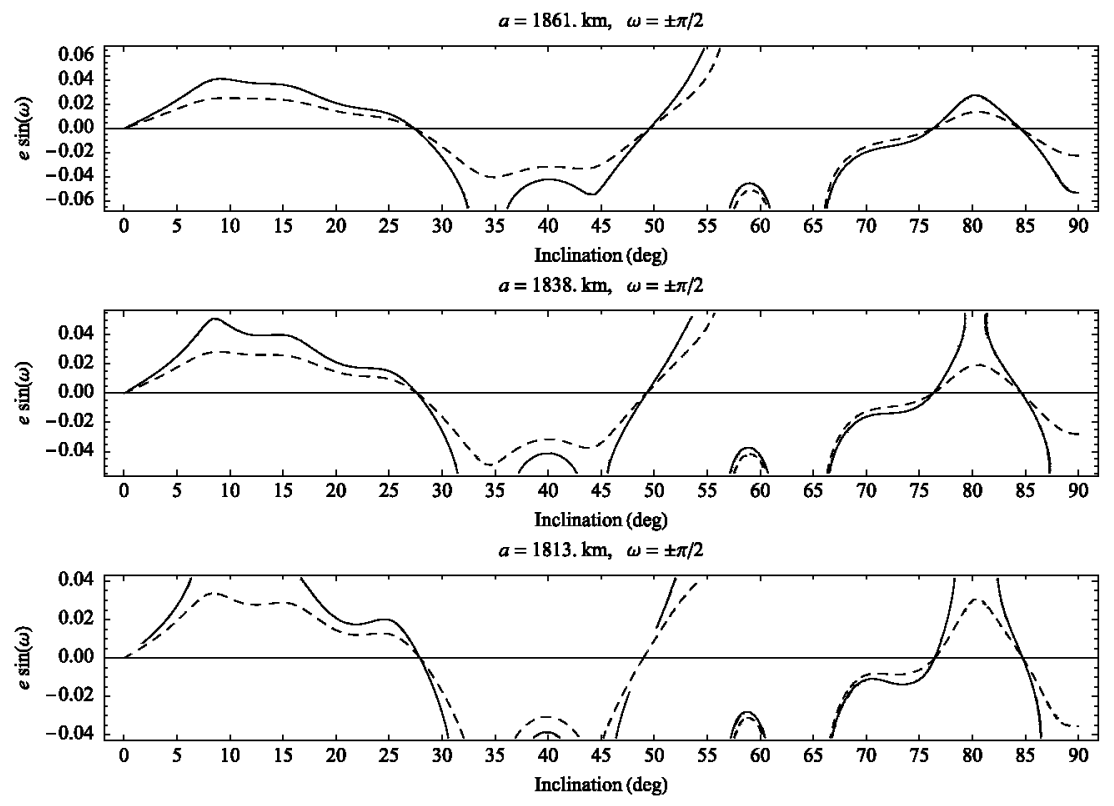

Fig. 6. Inclination-eccentricity diagrams of low lunar frozen orbits. Full line: natural dynamics. Dashed line: modified dynamics using a FRIT parallel to the Moon's equatorial plane, with a physical length $\lambda=50 \mathrm{~km}$ (characteristic length $\ell=17.7 \mathrm{~km}$.)

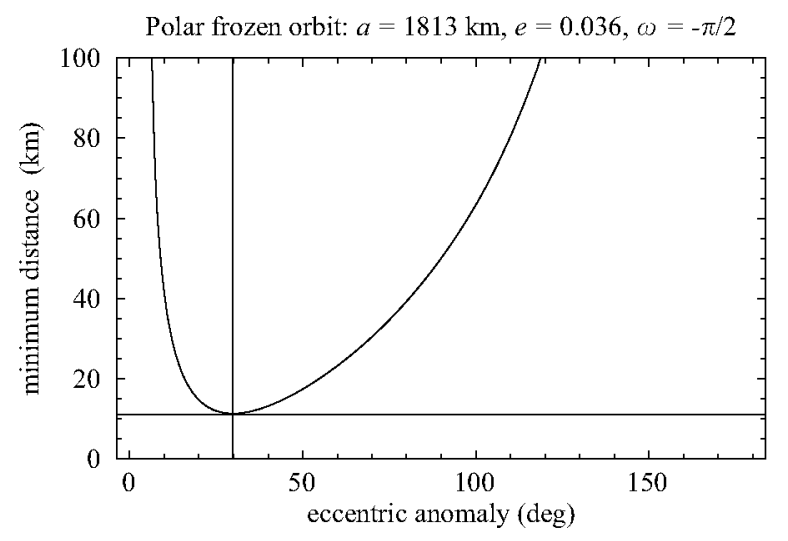

Fig. 7. Evolution of the minimum distance $\Delta$ to the lunar surface (as defined in Eq. (16)) of a FRIT of $50 \mathrm{~km}$ tether's length in the equal end masses configuration, which is in an elliptic polar frozen orbit about the Moon with semimajor axis $a=1813 \mathrm{~km}$.

symmetry with respect to polar introduced by the averaging procedure.

\section{Numerical validation}

The precedent results are based in several simplifications. Namely,

1. the gravitational perturbing forces and torques are truncated to the second order in the tether's length, Eqs. (2) and (5),

2. the equations were averaged over the tether's rotation angle, and

3. second order effects on the attitude dynamics were neglected, which are known to cause the tether's plane of rotation to precess.

To check the significance of these results, we study the dynamical evolution of some test cases in more realistic scenarios. In our comparisons, we used a numerical propagator that allows for the simultaneous integration of the tether's center of mass trajectory and its attitude. The model uses analytical ephemeris for the third-body perturbation and allows for choosing different subsets of the LP150Q gravity model [23]. A full description of the numerical propagator is given in Ref. [9],

Similar results have been obtained in all the cases tested, in which we tried four different configurations

Mass Point Untethered satellite, 50 zonal harmonics and Earth perturbations.

Equatorial Tether 1 Tether maintained in the oblate configuration, 150 zonal harmonics and Earth perturbations.

Equatorial Tether 2 Tether maintained in the oblate configuration, $50 \times 50$ harmonics and Earth perturbations.

Precessing Tether Tether initially in the oblate configuration but allowed to freely precess, 50 zonal harmonics and Earth perturbations.

Remark that a control torque must be applied in order to keep the tether in the oblate configuration-a choice that is allowed by the numerical simulator.

Fig. 8 shows one of the examples run. We choose the orbit with $a=1838 \mathrm{~km}$, and $i=81^{\circ}$ that is frozen in the oblate configuration using a FRIT of $50 \mathrm{~km}$ but it is not in the zero-length case-a mass point satellite, c.f. the center plot of Fig. 6. 


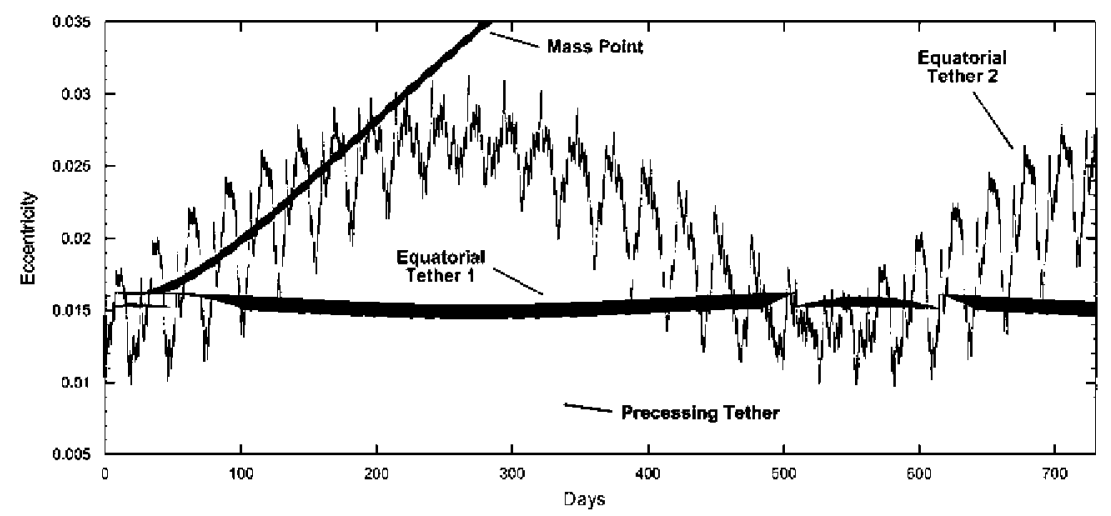

Fig. 8. Eccentricity evolution of a $50 \mathrm{~km} \mathrm{FRIT}\left(\Omega_{\perp}=0.1 \mathrm{rad} / \mathrm{s}\right)$ for a low-altitude $(a=1838 \mathrm{~km})$, high-inclination orbit $\left(i=81^{\circ}\right)$, using different dynamical models. The epoch is $\mathrm{J} 2000.0$

Thus, in reference to Fig. 8 , we check that the mass point case is not frozen, as expected, while the orbit eccentricity indeed remains bounded in all other cases, showing striking performances for the Equatorial Tether 1. These performances are mostly maintained by the (noncontrolled) Precessing Tether, with long-periodic excursions to almost circular orbits. The possible applications of such an orbit for observation missions, are clearly appealing. Finally, tesseral harmonics introduce short- and longperiod oscillations in the eccentricity of the Equatorial Tether 2.

As additional tests, we added the Sun's third body perturbation to all the cases analyzed. The most notable effects of this perturbation are monthly oscillations that remain anyway bounded.

\section{Conclusions}

Tether applications for modifying the orbital dynamics of an artificial satellite mission have been proposed since the early tether studies. However, most of them were related to satellite missions to the Lagrangian points.

The formal analogy between the potential of an oblate central body and that of an inert tether suggests to search for tether configurations that artificially modify the oblateness perturbation of a central body. One of these configurations is found for fast rotating tethers whose rotation axis is perpendicular to the equatorial plane of the central body. In this case, tether's length variations can be employed as a purely dynamical control mechanism that allows, to some extent, to modify the perturbation exerted by an oblate central body upon a satellite.

In particular, low lunar circular frozen orbits are known to exist only in a handful of specific inclinations. However, the eccentricity of low lunar frozen orbits with other inclinations can be notably lowered using fast rotating inert tethers of few tens of $\mathrm{km}$. This application can be of real interest for lunar missions requiring highinclination low-altitude orbits, as is the case of most observation missions. Additionally, the fact that the end masses of a rotating tether get periodically closer to the surface of the Moon is another positive point that may be quite important for high-resolution mapping missions.
In practice, the frozen orbits calculated with these criteria require attitude control to maintain the oblate configuration. But allowing the tether to precess still leads to orbits with bounded eccentricity, which may even imply further advantages for certain applications, such as periodical long stays in nearly circular orbits.

\section{Acknowledgments}

We thank an anonymous reviewer for highlighting the usefulness that FRITs may have for high-resolution observation missions. Support from the Government of Spain through projects AYA 2009-11896 (ML) and AYA 201018796 (all authors) of Ministry of Science and Innovation is acknowledged.

\section{References}

[1] S.L. Coffey, A. Deprit, B.R. Miller, The critical inclination in artificial satellite theory, Celestial Mech. 39 (4) (1986) 365-406.

[2] S.L. Coffey, A. Deprit, E. Deprit, Frozen orbits for satellites close to an earth-like planet, Celestial Mech. Dyn. Astron. 59 (1) (1994) $37-72$.

[3] A.S. Konopliv, W.L. Sjogren, R.N. Wimberly, R.A. Cook, A. Vijayaraghavan, A High Resolution Lunar Gravity Field and Predicted Orbit Behavior, Paper AAS 93-622, August, 1993.

[4] A. Konopliv, S.W. Asmar, E. Carranza, W.L. Sjogren, D.N. Yuan, Recent gravity models as a result of the lunar prospector mission, Icarus 150 (1) (2001) 1-18.

[5] M. Lara, Design of long-lifetime lunar orbits: a hybrid approach, Acta Astronaut. 69 (2011) 186-199, http://dx.doi.org/10.1016/j. actaastro.2011.03.009.

[6] D. Folta, D. Quinn, Lunar Frozen Orbits, Paper AIAA 2006-6749, August, 2006.

[7] J. Peláez, M. Sanjurjo, F.R. Lucas, M. Lara, E.C. Lorenzini, D. Curreli, D.J. Scheeres, Dynamics and Stability of Tethered Satellites at Lagrangian Points, ESA/ESTEC Report, Ariadna ID 07/4201, 2008, 423 pp. Document ACT-RPT-MAD-ARI-07-4201-Tethers.pdf + downloadable from $\langle\mathrm{http}: / \mathrm{www}$.esa.int/gsp/ACT/doc/ARI/ $\rangle$.

[8] J. Peláez, M. Lara, C. Bombardelli, F.R. Lucas, M. Sanjurjo-Rivo, D. Curreli, E.C. Lorenzini, D.J. Scheeres, Periodic orbits of the Hilltether satellite problem originated from the collinear points, J. Guidance Control Dyn. 35 (1) (2012) 222-233, http://dx.doi.org/ 10.2514/1.53097.

[9] H. Urrutxua, Use of Rotating Space Tethers in the Exploration of Celestial Bodies, Master Thesis in Aerospace Engineering, ETSI Aeronáuticos, Technical University of Madrid (UPM), September, 2010. 
[10] D.J. Scheeres, M.D. Guman, B.F. Villac, Stability analysis of planetary satellite orbiters: application to the Europa orbiter, J. Guidance Control Dyn. 24 (4) (2001) 778-787.

[11] M. Lara, J.F. San-Juan, Dynamic behavior of an orbiter around Europa, J. Guidance Control Dyn. 28 (2) (2005) 291-297.

[12] J. Peláez, M. Lara, Dynamics of fast-rotating tethered satellites, Monogr. Real Acad. Cienc. Zaragoza 32 (2009) 75-84.

[13] R.W. Farquhar, The Control and Use of Libration-Point Satellites, NASA TR R-346, 1970 , pp. 89-102.

[14] R.W. Farquhar, Tether stabilization at a collinear libration point, J. Astronaut. Sci. 49 (1) (2001) 91-106.

[15] A.K. Misra, J. Bellerose, V.J. Modi, Dynamics of a tethered system near the Earth-Moon Lagrangian points, Adv. Astronaut. Sci. 109 (2002) 415-435.

[16] B. Wong, A.K. Misra, Dynamics of a Multi-tethered System Near the Sun-Earth Lagrangian Point, Paper AAS 2003-218, 2003.

[17] B. Wong, A.K. Misra, Dynamics of a Libration Point Multi-tethered System, Paper IAC-04-A.5.09, 2004.
[18] E. Cutting, G.H. Born, J.C. Frautnick, Orbit analysis for SEASAT-A, J. Astronaut. Sci. 26 (1978) 315-342.

[19] G.W. Rosborough, C. Ocampo, Influence of Higher Degree Zonals on the Frozen Orbit Geometry, Paper AAS 91-429, August, 1991.

[20] B.E. Shapiro, Phase plane analysis and observed frozen orbit for the Topex/Poseidon Mission, Adv. Astronaut. Sci. 91 (1995) 853-872.

[21] R.P. Russell, M. Lara, Long-lifetime lunar repeat ground track orbits, J. Guidance Control Dyn. 30 (4) (2007) 982-993.

[22] K.C. Howell, D.J. Grebow, Z.P. Olikara, Design Using Gauss' Perturbing Equations with Applications to Lunar South Pole Coverage, Paper AAS 07-143, February, 2007.

[23] R.B. Roncoli, Lunar Constants and Models Document, JPL D-32296, Jet Propulsion Laboratory, Pasadena, CA, September, 2005 (Section 2.1).

[24] M. Lara, S. Ferrer, B. De Saedeleer, Lunar analytical theory for polar orbits in a 50-degree zonal model plus third-body effect, J. Astronaut. Sci. 57 (3) (2009) 561-577. 